\title{
Gravity wave momentum flux spectra observed from satellite in the summertime subtropics: Implications for global modeling
}

\begin{abstract}
M. Ern $^{1}$ and P. Preusse ${ }^{1}$
Received 6 June 2012; revised 9 July 2012; accepted 9 July 2012; published 15 August 2012.

[1] The spectral distribution in terms of horizontal and vertical wavenumber is deduced for gravity wave (GW) momentum flux observations from space for the first time. The paper focuses on High Resolution Dynamics Limb Sounder (HIRDLS) observations in the subtropics and compares spectra over particularly strong deep convective forcing with a background inferred from regions of less momentum flux. The deep convective spectra are strongly enhanced at high horizontal wavenumbers over a wide range of vertical wavenumbers and phase speeds. The observations indicate that GWs generated by deep convection are an important contribution on top of the background spectrum due to other GW sources (at least about $20-30 \%$ on zonal average is from convective GWs). The implications for global modeling are discussed. Citation: Ern, M., and P. Preusse (2012), Gravity wave momentum flux spectra observed from satellite in the summertime subtropics: Implications for global modeling, Geophys. Res. Lett., 39, L15810, doi:10.1029/2012GL052659.
\end{abstract}

\section{Introduction}

[2] The spectral distribution of gravity wave (GW) momentum flux is a research topic of particular interest. Because of the small horizontal scales of GWs chemistry-climate models (CCMs) do not resolve the full spectrum of GWs, and the effect on the atmospheric background flow has to be parameterized by GW drag schemes. In most CCMs orographic waves are parameterized based on simplifying physical assumptions, while all other wave sources, such as deep convection, or spontaneous emission from jets, are subsumed in one nonorographic GW parameterization [e.g., Alexander et al., 2010, and references therein].

[3] Because of a lack of observational constraints the nonorographic GW launch spectrum often is assumed to be constant (i.e., independent of geolocation, season, and GW propagation direction), and the choice of source parameters is more or less a result of tuning the CCM to reproduce a realistic circulation pattern. Both source amplitude and spectral shape are highly relevant for the resulting vertical profile of momentum deposition (GW drag) obtained. The assumption of a fixed nonorographic GW source distribution has several disadvantages. For example, changes of the GW

\footnotetext{
${ }^{1}$ Institut für Energie- und Klimaforschung, Stratosphäre, Forschungszentrum Jülich, Jülich, Germany.

Corresponding author: M. Ern, Institut für Energie- und Klimaforschung, Stratosphäre, Forschungszentrum Jülich, D-52425 Jülich, Germany. (m.ern@fz-juelich.de)

(C)2012. American Geophysical Union. All Rights Reserved. 0094-8276/12/2012GL052659
}

sources in response to a changing climate (e.g., changes in deep convection, or in the frequency of storms) might be an important feedback process currently missing in climate simulations. Therefore, GW momentum flux parameterizations dedicated to specific source processes, such as storms and fronts or deep convection, are under development and being implemented in CCMs [e.g., Charron and Manzini, 2002; Song et al., 2007; Richter et al., 2010]. Still, in these parameterizations the spectral shape is based on simplifying assumptions that have influence on where the spectrum saturates and momentum is deposited. Observational constraints on the spectral distribution of GW momentum flux for the background spectrum and dedicated sources can help to make these parameterizations more realistic.

[4] Our study focuses on the summertime subtropics between $10^{\circ}$ and $30^{\circ}$ latitude, where observed localized maxima in GW variances or momentum fluxes have been attributed to GWs excited by deep convection [e.g., Ern et al., 2004, 2011; Wright and Gille, 2011; Gong et al., 2012]. These characteristic maxima are clearly visible in global distributions of HIRDLS GW momentum fluxes at $25 \mathrm{~km}$ altitude (Figure 1). Figure 1a shows the GW momentum flux distribution in the northern hemisphere, averaged over boreal summer (June-August) and the years 2005-2007; Figure 1b shows the same for the southern hemisphere, averaged over austral summer (December-February) and the years 2005/ 2006-2007/2008. In boreal summer (Figure 1a) momentum flux maxima due to deep convection in the subtropics are found at $\sim 20^{\circ} \mathrm{N}$ over the Gulf of Mexico, North Africa, and Southeast Asia. In austral summer (Figure 1b) momentum flux maxima are found at $\sim 20^{\circ} \mathrm{S}$ over South America, South Africa, and North Australia.

[5] In section 2 instrument and data processing are briefly described. The resulting GW spectra are discussed in section 3; conclusions are given in section 4.

\section{Instrument and Analysis Method}

\subsection{The Satellite Instrument HIRDLS}

[6] Our study is based on version V006 temperature data of the HIRDLS instrument onboard the EOS-Aura satellite. Atmospheric temperatures are derived from the $15 \mu \mathrm{m}$ emission of $\mathrm{CO}_{2}$ in limb viewing geometry. HIRDLS observations cover the time period from January 2005 to March 2008. With a latitudinal coverage of $63^{\circ} \mathrm{S}$ to $80^{\circ} \mathrm{N}$ observations are near-global. The altitude range covered is $\sim 10-75 \mathrm{~km}$. The random temperature error is $\sim 0.5 \mathrm{~K}$ at $20 \mathrm{~km}$ altitude. The error of GW amplitudes determined in our study is at least a factor of two better owing to vertical averaging (see below). More information about the HIRDLS 

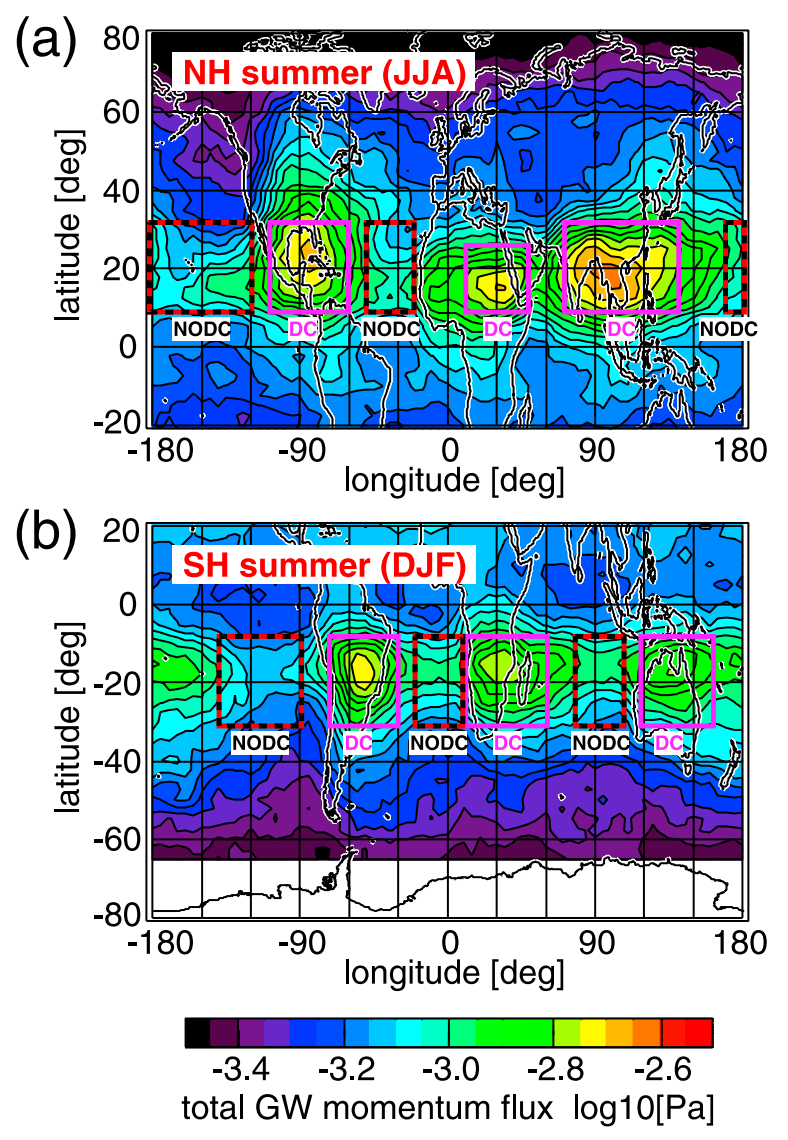

Figure 1. Horizontal distribution of HIRDLS total GW momentum flux on a logarithmic scale in (a) the northern and (b) the southern hemisphere at $25 \mathrm{~km}$ altitude during summertime. Values are averaged over each full summer period of the respective hemisphere (June to August for the northern, and December to February for the southern hemisphere) and all years covered by HIRDLS observations.

instrument and temperature retrieval can be found, for example, in Gille et al. [2008, and references therein].

\subsection{Observed Gravity Wave Momentum Flux}

[7] Total GW momentum flux is derived from subsequent pairs of HIRDLS temperature altitude profiles along the satellite measurement track [Ern et al., 2011]. The largescale atmospheric background for zonal wavenumbers up to 6 is estimated by two-dimensional spectral decomposition in longitude and time and subtracted [Ern et al., 2011]. From the residual temperatures, GW amplitudes, phases and vertical wavelengths for the strongest wave component in each altitude profile are determined for moving $10 \mathrm{~km}$ vertical windows [Preusse et al., 2002]. Horizontal wavelengths are determined from vertical phase shifts between pairs of altitude profiles. These values are however the apparent horizontal wavelength along the satellite measurement track and will usually overestimate the true value. The ranges of GW vertical and horizontal wavelengths covered by the method are limited by the observational filter of the instrument [e.g., Preusse et al., 2002, 2009; Alexander et al., 2010] and the analysis method. Our study covers vertical wavelengths of $2-25 \mathrm{~km}$ and horizontal wavelengths $>100-200 \mathrm{~km}$ [see Ern et al., 2011]. Since pairs of altitude profiles provide only 2D information, no information about the propagation direction of GWs is obtained and only total momentum flux (i.e., absolute values) can be determined via equation (7) of Ern et al. [2004]. Uncertainties of these values are large, at least a factor of two [e.g., Ern et al., 2004, 2011].

\subsection{Estimation of Gravity Wave Momentum Flux Spectra}

[8] It has been shown by Lehmann et al. [2012] that the GW momentum flux spectrum in a region can be recovered by sampling the wave field at many locations. This method has been tested for three dimensions, but it demonstrates that in general spectra can be retrieved by integrating over a multitude of single-wave fits in a sampled volume. This applies to both model data and observations by various instruments. However the observational limitations have to be carefully considered. Current limb scanning instruments like HIRDLS are limited to a single measurement track providing only 2D information on the observed GWs. For these data GW momentum flux spectra represent the spectral information averaged over the whole azimuth of GW propagation directions.

[9] GW momentum flux spectra are determined from single HIRDLS observations (with their horizontal and vertical wavelengths $\lambda_{h}(i)$ and $\lambda_{z}(i)$ ) by binning them in the plane of horizontal $k_{h}$ and vertical wavenumbers $m\left(k_{h}=2 \pi / \lambda_{h}\right.$ and $m=2 \pi / \lambda_{z}$ ). For this purpose, we calculate for each observation the new coordinates $\widetilde{k}_{h}(i)=\log _{10}\left(1 / \lambda_{h}(i)\right), \widetilde{m}(i)=$ $\log _{10}\left(1 / \lambda_{z}(i)\right)$, and define an equispaced grid in these coordinates with grid spacings of $\delta \widetilde{k_{h}}=0.1$ and $\delta \widetilde{m}=0.1$. The single observations are then sorted into overlapping square bins of width $\Delta \widetilde{k_{h}}=0.2$ and $\Delta \widetilde{m}=0.2$, centered at the grid points. Momentum fluxes are added for each bin separately and attributed to the corresponding grid point. The resulting spectrum is normalized such that the integral over the spectrum results in the average momentum flux of all observations used for estimating the spectrum.

[10] Errors of vertical wavelengths are random [Preusse et al., 2002] and will average out due to the large number of single observations considered. Also undersampling of observed GWs will not affect our results because the HIRDLS horizontal sampling step of $\sim 100 \mathrm{~km}$ allows to resolve most of the GWs observed. Since HIRDLS observes only the projection of the true GW horizontal wavelength on the measurement track, horizontal wavenumbers will be lowbiased [Preusse et al., 2009]. This will result in a low-bias of momentum fluxes in all spectra shown, as well as a slight systematic shift of the spectra toward low horizontal wavenumbers $(\sim 0.15$ in units of the logarithmic $x$-axes in Figure 2). This will however likely not affect the main results of our study. The most important error source is the instrument sensitivity function, as discussed in section 3 .

\section{Gravity Wave Momentum Flux Spectra in the Summertime Subtropics}

[11] We subdivide the subtropical latitude bands between $10^{\circ}$ and $30^{\circ}$ in both hemispheres into longitude/latitude regions that are likely to be dominated by strong convective GW activity during summertime. These "deep convection" (DC) regions are indicated by magenta rectangles in Figure 1. As a reference, we also define "control" (NoDC) regions that 

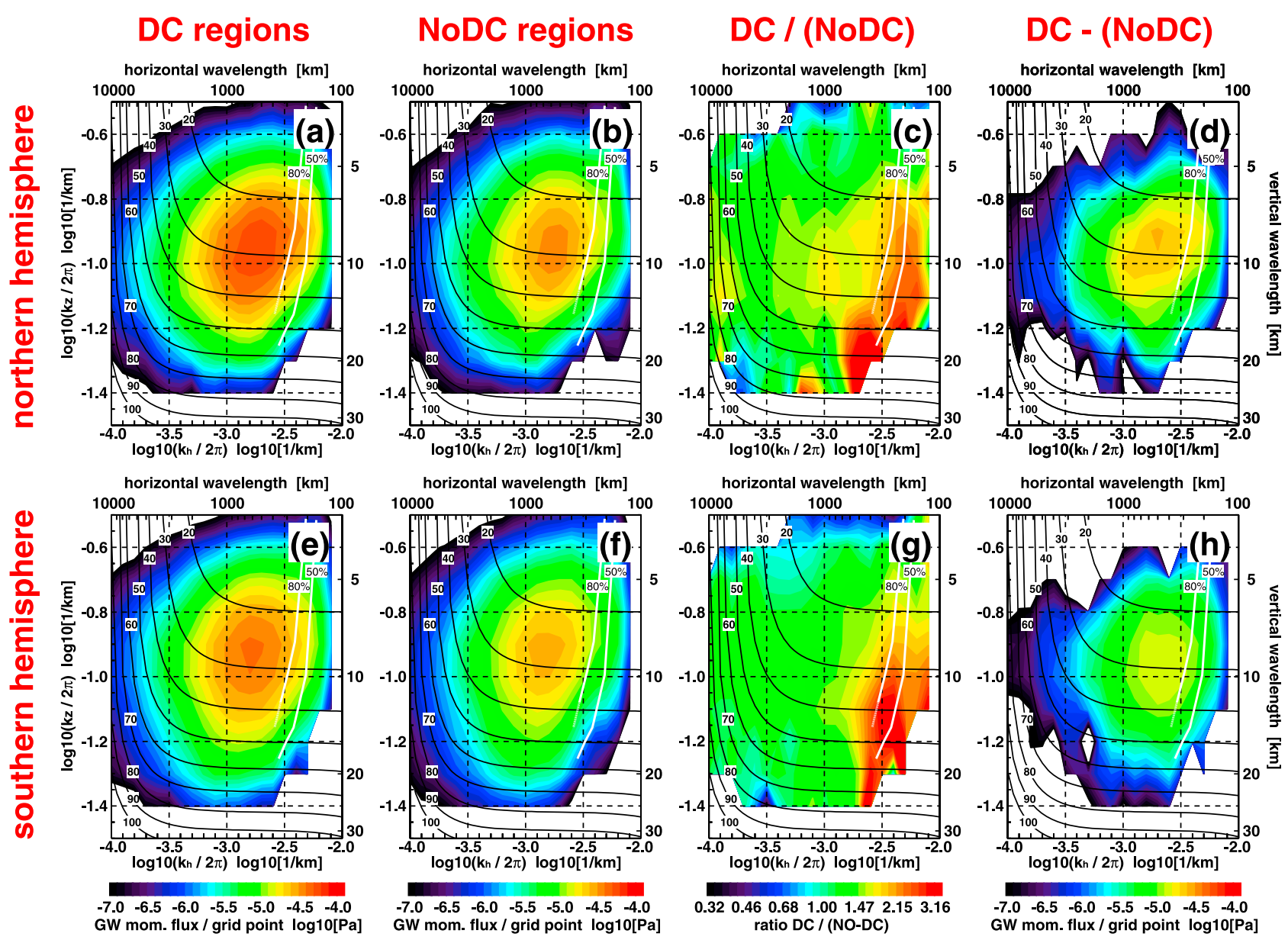

Figure 2. GW momentum flux spectra in the summertime subtropics at $25 \mathrm{~km}$ altitude plotted as function of horizontal $\left(k_{h}\right)$ and vertical $\left(k_{z}\right)$ wavenumber on logarithmic scales. Shown are, for the northern hemisphere, the spectrum averaged over (a) the three "deep convective" (DC) regions defined in Figure 1a, and (b) the regions less dominated by convective GWs (NoDC) (see Figure 1a). Also shown are (c) the ratio, and (d) the difference of the spectra shown in Figures 2a and 2b. $(\mathrm{e}-\mathrm{h})$ The same as Figures $2 \mathrm{a}-2 \mathrm{~d}$, but for the southern hemisphere and the regions defined in Figure 1b. Again, values are averages over each full summer period of the respective hemisphere and all years covered by HIRDLS observations. Black contour lines given in all panels indicate intrinsic phase speeds in $\mathrm{m} / \mathrm{s}$. White contour lines indicate the sensitivity of infrared limb sounders to the momentum flux of GWs with given horizontal and vertical wavelength [cf. Ungermann et al., 2010, Figure 14].

are likely less dominated by deep convection (red-black dashed rectangles in Figure 1). For each region the average momentum flux spectrum is estimated for every full summer period of the respective hemisphere (December to February in the southern, and June to August in the northern hemisphere) covered by HIRDLS data. For each hemisphere average DC (NoDC) spectra are obtained by averaging over all available full summer periods and by combining also the spectra of the different DC (NoDC) regions in each hemisphere.

[12] The resulting average spectra are given in Figure 2. Shown are the DC spectrum for the northern hemisphere (Figure 2a), the NoDC spectrum (Figure $2 b$ ), the ratio DC/ NoDC (Figure 2c), and the difference DC minus NoDC (Figure $2 \mathrm{~d}$ ). Figures $2 \mathrm{e}-2 \mathrm{~h}$ show the same, but for the southern hemisphere. All spectra (Figures 2a, 2b, 2e, and 2f) have common characteristics and display spectral distributions with a spectral peak at about $600 \mathrm{~km}$ horizontal and $10 \mathrm{~km}$ vertical wavelength, and a drop in the spectrum to all sides. The vertical wavenumber position of the spectral peak should be fairly robust, because the peak is well inside the observational range of the instrument and the vertical wavelength range covered by the analysis method. The horizontal wavenumber position of the spectral peak is somewhat low-biased, because the sensitivity of a limbscanning instrument starts to decrease at horizontal wavelengths shorter than $\sim 600 \mathrm{~km}$ [see also Preusse et al., 2002]. Because low amplitude GWs are less likely detected by our analysis method, the decrease of the spectrum at low momentum fluxes will be generally exaggerated. However, in the vicinity of the spectral peak this effect remains small [Lehmann et al., 2012].

[13] The ratio of DC and NoDC spectra (Figures $2 \mathrm{c}$ and $2 \mathrm{~g}$ ) reveals that at low and medium horizontal wavenumbers the momentum flux of DC and NoDC spectra is comparable, or only somewhat higher in the DC spectra. At high horizontal wavenumbers, however, the DC spectra are strongly enhanced (at $\mathrm{x}$-axis values $>-2.9 \ldots-2.8$, i.e., $\lambda_{h}<600 \ldots$ 
$800 \mathrm{~km}$ ). This enhancement is found for a large intrinsic frequency range of $20-70 \mathrm{~m} / \mathrm{s}$ (see the black solid contour lines overplotted in all panels of Figure 2). This enhancement is an effect of the deep convective sources that are not contained or less frequent in the NoDC regions and that are expected to contribute also at short horizontal wavelengths.

[14] If we assume that the NoDC spectrum is mainly the result of unspecified GW sources (shallower convection, wind shear,...) that are also present in the DC regions, this would explain the similarity of DC and NoDC spectral shapes at low and medium horizontal wavenumbers. Because deep convection is very intermittent in nature, the convective GWs additionally contained in the DC spectra will not be present the whole time, and therefore they will not completely mask the NoDC-like part of the spectrum. Our finding of a non-convective GW background spectrum is qualitatively in good agreement with the model study of convective GWs by Choi et al. [2012]. In this study additional background GWs were required to obtain results comparable to observations by Gong et al. [2012].

[15] The difference between DC and NoDC spectra shown in Figures $2 \mathrm{~d}$ and $2 \mathrm{~h}$ can be interpreted as the additional spectral contribution of the deep convective sources in the DC regions of the northern and southern hemisphere, respectively (negative values are mostly insignificant and therefore not plotted). Assuming a typical average background wind of $10-20 \mathrm{~m} / \mathrm{s}$ in the summertime lower stratosphere at $\sim 25 \mathrm{~km}$ altitude and GW propagation prevalently opposite to these winds, the location of the spectral peak around $30 \mathrm{~m} / \mathrm{s}$ intrinsic phase speed (see Figures $2 \mathrm{~d}$ and $2 \mathrm{~h}$ ) is broadly consistent with parameterized and simulated convective GW spectra peaking at ground based phase speeds of about $10 \mathrm{~m} / \mathrm{s}$ at the cloud top [e.g., Choi and Chun, 2011], if the Doppler shift of the spectrum is taken into account.

[16] Also shown in all panels of Figure 2 are two white contour lines, indicating where the sensitivity of infrared limb sounders to GW squared amplitudes or momentum fluxes has decreased to $80 \%$ and $50 \%$, respectively [cf. Ungermann et al., 2010, Figure 14]. The expected loss of sensitivity between the $80 \%$ and $50 \%$ contour lines corresponds to about two color levels of the logarithmic momentum flux scales in Figure 2. In the DC and NoDC spectra the observed momentum flux decrease between peak position and the $50 \%$ contour line is always stronger than this, and the position of the spectral peak is resolved in both horizontal and vertical wavelength. In Figures $2 \mathrm{~d}$ and $2 \mathrm{~h}$ the decrease between spectral peak and 50\%-line is weaker. Nevertheless, it is indicated that the "true" spectrum flattens out, some kind of peak is reached, and large part of this spectral contribution of the overall convective spectrum is detected. Further spectral peaks or enhancements of the convective spectrum that may be located at even shorter horizontal wavelengths and would correspond to source processes proposed by Fovell et al. [1992] or Lane et al. [2001] are however outside the observational range of HIRDLS.

[17] In the northern hemisphere the total momentum flux in Figure $2 \mathrm{a}$ (DC case) is $1.79 \mathrm{mPa}$. Out of this, $1.24 \mathrm{mPa}$ are at $\lambda_{h}<800 \mathrm{~km}$, and $0.55 \mathrm{mPa}$ at $\lambda_{h}>800 \mathrm{~km}$. In the NoDC case the corresponding values are $1.08,0.71$, and $0.37 \mathrm{mPa}$, respectively. In the southern hemisphere corresponding values in the $\mathrm{DC}$ case are $1.43,0.95$, and $0.48 \mathrm{mPa}$, respectively, and $0.96,0.58$, and $0.38 \mathrm{mPa}$ in the NoDC case. This means that enhanced total momentum flux in the DC regions is mainly due to the additional contribution of convective GWs at horizontal wavelengths $200-800 \mathrm{~km}$. The ratio of momentum fluxes at $\lambda_{h}<800 \mathrm{~km}$ and $\lambda_{h}>800$ $\mathrm{km}$ is for all cases between about 1.5 and 2.3. In the DC cases the ratio is about $20-30 \%$ higher than in the NoDC cases of the respective hemisphere.

[18] Now we assume that the momentum flux contained in the NoDC regions is representative of the non-deepconvective GW spectrum in the whole latitude bands considered. Zonal averages in absence of deep convection would be $1.08 \mathrm{mPa}(0.96 \mathrm{mPa})$ in the northern (southern) subtropics, i.e., the total momentum fluxes of the NoDC regions (see above). Overall zonal averages in these latitude bands are however $1.42 \mathrm{mPa}(1.18 \mathrm{mPa})$ for the northern (southern) hemisphere, i.e., $31 \%(23 \%)$ higher. These percentages are likely lower estimates for the additional contribution due to convective GWs because also the NoDC regions will still contain some deep convection. Wright and Gille [2011] find momentum flux enhancements of $\sim 20-50 \%$ (typically $\sim 30 \%$ ) in the monsoon regions during summer. Their values are a little higher than our results. However, since different from our study wintertime values are used as a reference, they attribute part of their enhancement to reasons other than deep convection, i.e., different meteorological conditions.

\section{Summary and Discussion}

[19] Gravity wave (GW) momentum flux spectra averaged over all azimuths are derived from temperature observations of the HIRDLS satellite instrument for the summer subtropics. By comparing GW spectra over deep convection and reference regions, the spectral contribution of GWs excited by deep convection is estimated. The spectral peak for convective waves is at shorter horizontal wavelengths $\left(\lambda_{h} \approx\right.$ $500 \mathrm{~km})$ than in the reference spectra. The contribution of convective waves covers a broad range of intrinsic phase speeds and will therefore interact with the atmospheric background winds at various altitudes. However, since the momentum flux decrease at short horizontal wavelengths is close to the expected loss of instrument sensitivity, it is indicated that part of the convective GW momentum flux is not measured.

[20] Limb sounders can only observe GWs with intrinsic periods $>1$ hour [Alexander et al., 2010], and they would only be able to observe a small tail of the convective GWs generated by the mechanical oscillator mechanism that involves time scales as short as $20 \mathrm{~min}$ [e.g., Fovell et al., 1992; Lane et al., 2001; Choi et al., 2012]. The time scales of the convective GWs identified in our study are $~$ hours, compliant with the lifetime of convective systems [Pfister et al., 1993], maybe indicating a different source mechanism.

[21] Convective GWs of a few hundred kilometer horizontal wavelengths are particularly difficult to model as both large domain and high resolution for the convective processes are required. Observational constraints are therefore important, in particular, since our observations indicate that already the contribution of convective GWs visible for infrared limb sounders is at least $20-30 \%$ of the observed GW momentum flux in the subtropics. 
[22] Acknowledgments. This work benefitted from discussions at the International Space Science Institute (ISSI), Bern. M. Ern was supported by Deutsche Forschungsgemeinschaft (DFG) grant ER 474/2-1. HIRDLS data were provided by NASA.

[23] The Editor thanks Jie Gong and an anonymous reviewer for assisting in the evaluation of this paper.

\section{References}

Alexander, M. J., et al. (2010), Recent developments in gravity-wave effects in climate models and the global distribution of gravity-wave momentum flux from observations and models, $Q$. J. R. Meteorol. Soc., 136, 1103-1124, doi:10.1002/qj.637.

Charron, M., and E. Manzini (2002), Gravity waves from fronts: Parameterization and middle atmosphere response in a general circulation model, J. Atmos. Sci., 59, 923-941.

Choi, H.-J., and H.-Y. Chun (2011), Momentum flux spectrum of convective gravity waves. Part I: An update of a parameterization using mesoscale simulations, J. Atmos. Sci., 68, 739-759.

Choi, H.-J., H.-Y. Chun, J. Gong, and D. L. Wu (2012), Comparison of gravity wave temperature variances from ray-based spectral parameterization of convective gravity wave drag with AIRS observations, J. Geophys. Res., 117, D05115, doi:10.1029/2011JD016900.

Ern, M., P. Preusse, M. J. Alexander, and C. D. Warner (2004), Absolute values of gravity wave momentum flux derived from satellite data, J. Geophys. Res., 109, D20103, doi:10.1029/2004JD004752.

Ern, M., P. Preusse, J. C. Gille, C. L. Hepplewhite, M. G. Mlynczak, J. M. Russell III, and M. Riese (2011), Implications for atmospheric dynamics derived from global observations of gravity wave momentum flux in stratosphere and mesosphere, J. Geophys. Res., 116, D19107, doi:10.1029/ 2011JD015821.

Fovell, R., D. Durran, and J. R. Holton (1992), Numerical simulations of convectively generated stratospheric gravity waves, J. Atmos. Sci., 49, $1427-1442$

Gille, J. C., et al. (2008), High Resolution Dynamics Limb Sounder: Experiment overview, recovery, and validation of initial temperature data, J. Geophys. Res., 113, D16S43, doi:10.1029/2007JD008824.
Gong, J., D. L. Wu, and S. D. Eckermann (2012), Gravity wave variances and propagation derived from AIRS radiances, Atmos. Chem. Phys., 12, 1701-1720

Lane, T. P., M. J. Reeder, and T. L. Clark (2001), Numerical modeling of gravity wave generation by deep tropical convection, J. Atmos. Sci., 46, 1249-1274.

Lehmann, C. I., Y.-H. Kim, P. Preusse, H.-Y. Chun, M. Ern, and S.-Y. Kim (2012), Consistency between Fourier transform and small-volume fewwave decomposition for spectral and spatial variability of gravity waves above a typhoon, Atmos. Meas. Tech., 5, 1637-1651, doi:10.5194/amt-51637-2012.

Pfister, L., S. Scott, M. Loewenstein, S. Bowen, and M. Legg (1993), Mesoscale disturbances in the tropical stratosphere excited by convection: Observations and effects on the stratospheric momentum budget, J. Atmos. Sci., 50, 1058-1075.

Preusse, P., A. Dörnbrack, S. D. Eckermann, M. Riese, B. Schaeler, J. T. Bacmeister, D. Broutman, and K. U. Grossmann (2002), Space-based measurements of stratospheric mountain waves by CRISTA: 1. Sensitivity, analysis method, and a case study, J. Geophys. Res., 107(D23), 8178, doi:10.1029/2001JD000699.

Preusse, P., S. Schroeder, L. Hoffmann, M. Ern, F. Friedl-Vallon, H. Oelhaf, H. Fischer, and M. Riese (2009), New perspectives on gravity wave remote sensing by spaceborne infrared limb imaging, Atmos. Meas. Tech., 2, 299-311.

Richter, J. H., F. Sassi, and R. R. Garcia (2010), Toward a physically based gravity wave source parameterization in a general circulation model, J. Atmos. Sci., 67, 136-156.

Song, I.-S., H.-Y. Chun, R. R. Garcia, and B. A. Boville (2007), Momentum flux spectrum of convectively forced internal gravity waves and its application to gravity wave drag parameterization. Part II: Impacts in a GCM (WACCM), J. Atmos. Sci., 64, 2286-2308.

Ungermann, J., L. Hoffmann, P. Preusse, M. Kaufmann, and M. Riese (2010), Tomographic retrieval approach for mesoscale gravity wave observations by the PREMIER Infrared Limb-Sounder, Atmos. Meas. Tech., 3, 339-354.

Wright, C. J., and J. C. Gille (2011), HIRDLS observations of gravity wave momentum fluxes over the monsoon regions, J. Geophys. Res., 116, D12103, doi:10.1029/2011JD015725. 